\title{
Successful Management of Penetrating Cardiac Injury: Case Report
}

\section{Carlos Pacheco Molina ${ }^{1 *}$, Héctor Vergara Miranda ${ }^{1}$, Oscar Rene García de León ${ }^{1}$, Ilse Andrea Moreno Arquieta ${ }^{2}$ and Gerardo Enrique Muñoz Maldonado ${ }^{1}$}

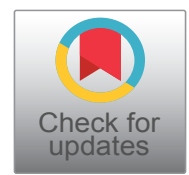

${ }^{1}$ Surgery Department, Autonomous University of Nuevo Leon, Mexico

${ }^{2}$ Medical School, Autonomous University of Nuevo Leon, Mexico

*Corresponding author: Carlos Pacheco Molina, Surgery Department, Autonomous University of Nuevo Leon, Dr. José Eleuterio González S/N, Mitras Centro, 64460 Monterrey, Nuevo León, México

\section{Keywords \\ Emergency thoracotomy, Cardiac injury, Cardiac surgery}

\section{Introduction}

Penetrating Cardiac Injury $(\mathrm{PCl})$ is a rare but life-threatening clinical condition in trauma patients. According to previous reports, incidence of $\mathrm{PCl}$ admissions to trauma centers has been reported to be of $0.16 \%$, with heterogeneous survival rates being as low as 3\% and as high as $84 \%$ [1]. This type of injury is a clinical challenge for surgeons due to its short therapeutic window and the fact that its treatment is dictated by the underlying mechanism of injury and hemodynamic status of the patient [2]. There are three major disorders associated with $\mathrm{PCl}$, these being hemorrhage, cardiac tamponade and cardiac failure, being cardiac tamponade an early opportunity for survival; however, also contributing very rapidly to mortality associated with cardiac wounds [3]. These factors translate into a personalized and immediate surgical approach, which is the only alternative to preserve the life of the patient.

\section{Case Description}

A 17-year-old male was admitted to the emergency room after receiving a chest injury by a sharp object; the wound, of approximately 3 centimeters, was located in the precordial region of the left hemitorax, at the ninth intercostal space on the clavicular midline (Figure 1).

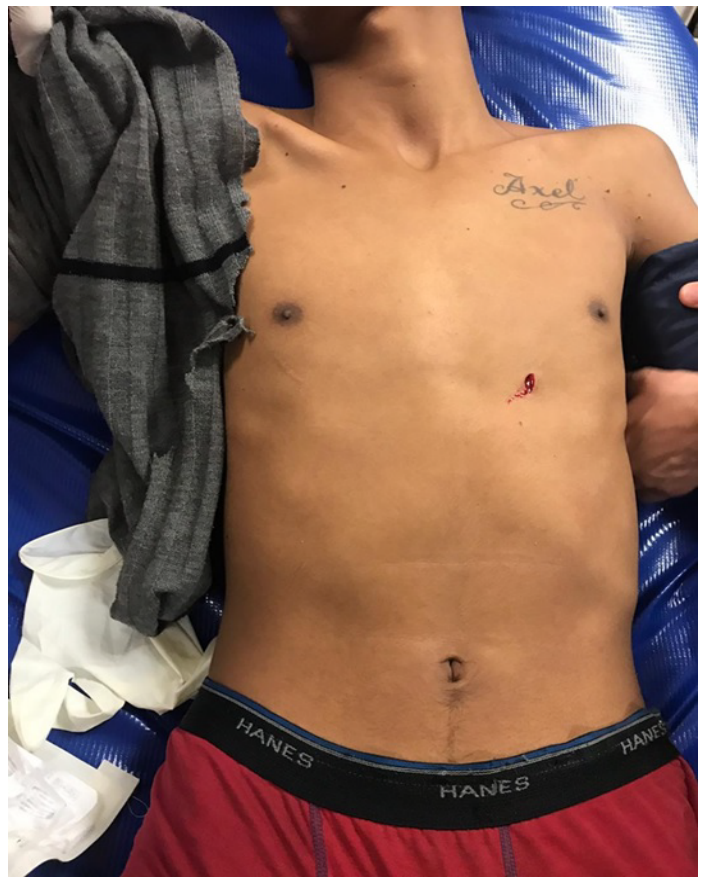

Figure 1: Location of the wound in the precordial region, in the left hemitorax in the ninth intercostal space at the level of the clavicular midline.

On his initial assessment, he presented the following vital signs: blood pressure of 100/70 mmHg, 104 cardiac beats per minute and 25 breaths per minute. Crystalloid solutions were administrated immediately (Hartmann 1000 milliliter solution), physical examination revealed adequately ventilated lungs, presence of agement of Penetrating Cardiac Injury: Case Report. Trauma Cases Rev 6:082. doi.org/10.23937/24695777/1510082 


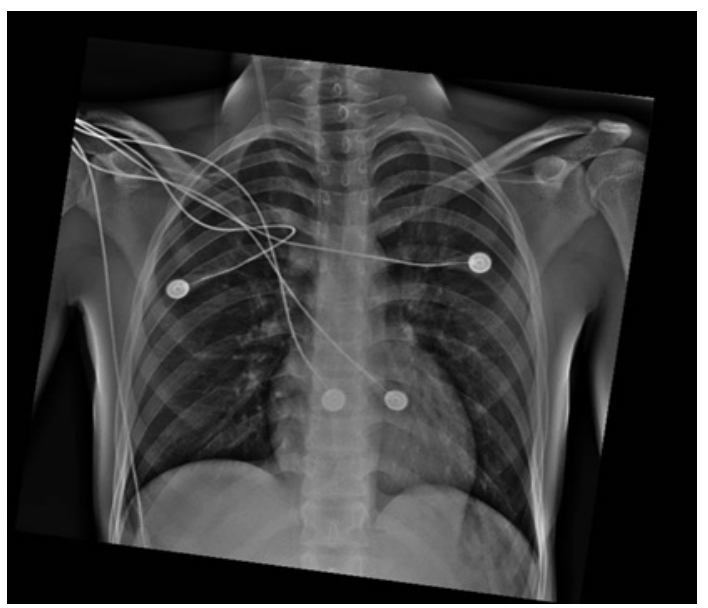

Figure 2: Chest X-ray without findings of pneumothorax, hemothorax or mediastinal enlargement.

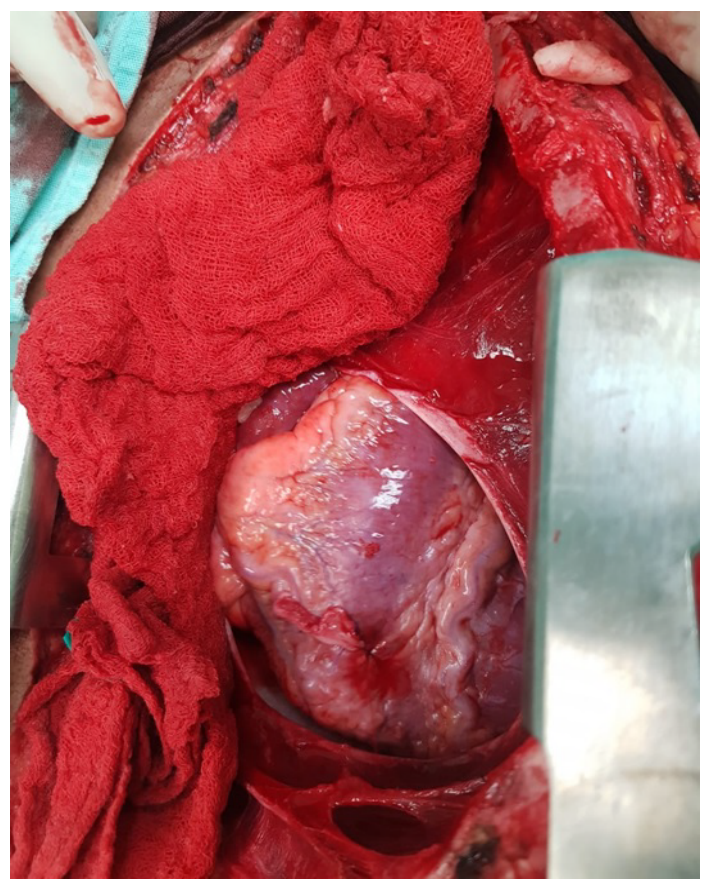

Figure 3: Pericardial window, showing the cardiac injury of $1 \mathrm{~cm}$ of length in the right ventricle grade IV.

heart sounds and no evidence of cardiac tamponade. Based on his clinical data and hemodynamic status, it was decided to perform complementary studies. Chest $x$-ray had no findings of pneumothorax, hemothorax or mediastinal enlargement (Figure 2), and transthoracic echocardiography was inconclusive.

A surgical approach via a subxiphoid pericardial window was decided. The subxiphoid incision was performed, dissecting to approach fascia, boarding preperitoneal space until identifying the membranous diaphragm and pericardium where the pericardial window resulted positive with findings of hemopericardium. Furthermore, a middle sternotomy was performed, incising the pericardium longitudinally with a finding of hemopericardium of 100 milliliters and a cardiac injury of $1 \mathrm{~cm}$ of length in the right ventricle grade IV (according to OIS- ASST system). To achieve temporary bleeding control, digital

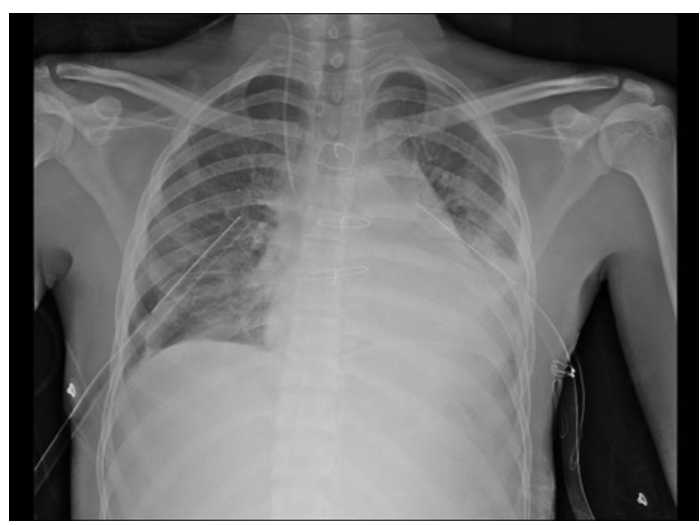

Figure 4: Chest x-ray post-surgery, were we can see the Blake drain and the two chest tubes placed bilaterally in the posterior pleural space.

direct pressure was carried out and for definitive repair, a primary closure of the injury was performed with 3-0 polypropylene U-point suture (Figure 3 and Video 1 ), covering it with a pericardial patch. Immediately after, a sternum closure with surgical wire was performed with the placement of a $19 \mathrm{Fr}$ Blake drain in the mediastinum, followed by two others $28 \mathrm{Fr}$ drains. Chest tubes were placed bilaterally in the posterior pleural space (Figure 4). The patient had immediate post-surgical stability, was successfully extubated and transferred to intermediate care unit.

In his post-operative period, patient remained hemodynamically stable with a favorable course, no inflammatory data or respiratory compromise was present, tolerated oral diet, thorax had no evidence of alterations, cardiac noises were present and with no presence of abnormal clinical data or evidence of cardiac tamponade. Broad-spectrum antibiotics were administrated due to mediastinitis risk, and a minimum quantity of serohematic fluid was obtained from the drains which were later withdrawn without further complications; he was discharged after 7 days of hospitalization.

\section{Discussion}

Initial head-to-toe systematic examination and awareness of the path of the penetrating object are important. Early establishment of an airway, restoration of the intravascular volume, evaluation of pneumothorax, hemothorax, hemopneumothorax with or without tension, cardiac tamponade data and assessment of the open chest wounds are the immediate objectives in the patient with a penetrating thoracic injury [4] Sometimes complementary studies may be helpful in the preoperative diagnose, being the ultrasound the most widely practiced method, however is both operator and patient dependent which make it fallible and can make the diagnosis highly deceptive.

Surgical and diagnostic approach are fundamental in the primary step to control the bleeding, the procedure of choice was the pericardial window with a sternotomy as the surgical access. It is known that median ster- 
notomy is the best approach, since it gives an effective and extensive vision of heart, great vessels, to other structures in the mediastinum, to both pleural cavities and can be performed rapidly, which is preferable in situations where traumatic hemopericardium has been confirmed on preoperative ultrasound [5], however like it was mentioned before in this case we did not have a preoperative conclusive diagnose. Nevertheless, the pericardial window and sternotomy were a satisfactory diagnostic and surgical approach respectively and had a favorable post-operative course.

\section{Conclusion}

Despite the limitations of the complementary studies the possibility of cardiac injury must be kept in patients with penetrating thoracic injury. The pericardial window is an accurate procedure for diagnose and surgical intervention with a quick management is imperative for the survival of the patient.

\section{References}

1. Isaza-Restrepo A, Bolívar-Sáenz DJ, Tarazona-Lara M, Tovar JR (2017) Penetrating cardiac trauma: Analysis of 240 cases from a hospital in Bogota, Colombia. World $\mathrm{J}$ Emerg Surg 12: 26.

2. Mishra B, Gupta A, Sagar S, Singhal M, Kumar S (2016) Traumatic cardiac injury: Experience from a level-1 trauma center. Chin J Traumatol 19: 333-336.

3. Mohd Lateef Wani, Ab Gani Ahangar, Shadab Nabi Wani, Ifat Irshad, Nayeem Ul-Hassan (2012) Penetrating cardiac injury: A review. Trauma Mon 17: 230-232.

4. Mandal AK, Sanusi M (2001) Penetrating chest wounds: 24 years experience. World J Surg 25: 1145-1149.

5. Kang N, Hsee L, Rizoli S, Alison P (2009) Penetrating cardiac injury: Overcoming the limits set by nature. Injury 40 : 919-927. 